\title{
Green tea catechins prevent obesity through modulation of peroxisome proliferator-activated receptors
}

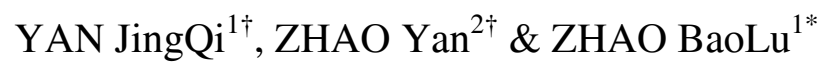 \\ ${ }^{1}$ State Key Laboratory of Brain \& Cognitive Sciences, Institute of Biophysics, Chinese Academy of Sciences, Beijing 100101, China; \\ ${ }^{2}$ Department of Food Sciences and Technology, Harbin Institute of Technology at Weihai, Weihai 264209, China
}

Received March 27, 2013; accepted May 24, 2013; published online July 10, 2013

\begin{abstract}
Epidemiological evidence and experimental studies suggest that drinking green tea is associated with a lower risk of obesity and related diseases. However, the mechanisms of these effects are not clear. In the present study, we investigated the antiobesity mechanisms of green tea catechins (GTCs) through modulation of peroxisome proliferator activated-receptor (PPAR) pathways in high-fat diet-induced obesity in rats. GTC supplementation significantly attenuated the increased body and liver weights and the elevated serum and liver triglyceride levels. Meanwhile, GTCs increased the PPAR $\gamma$ levels in subcutaneous white adipose tissue (SWAT) and decreased the PPAR $\gamma$ levels in visceral white adipose tissue (VWAT). In addition, GTC treatment up-regulated the levels of PPAR $\delta$ in SWAT, VWAT, and brown adipose tissue and increased the expression of genes involved in fatty acid oxidation in brown adipose tissue. Our results suggest that GTCs exert their anti-obesity mechanism in part by modulating PPAR signaling pathways.
\end{abstract}

obesity, green tea catechins, peroxisome proliferator activated-receptors (PPARs), hyperlipidemia, metabolic syndrome

Citation: Yan J Q, Zhao Y, Zhao B L. Green tea catechins prevent obesity through modulation of peroxisome proliferator-activated receptors. Sci China Life Sci, 2013, 56: 804-810, doi: 10.1007/s11427-013-4512-2

Obesity, a condition that increases the risk of a number of chronic diseases such as type 2 diabetes mellitus (T2DM), hypertension, hyperlipidemia and cardiovascular disease, has rapidly increased worldwide over the last few decades $[1,2]$. Epidemiological studies and experimental data show that drinking green tea or administration of its components, green tea catechins (GTCs), is associated with a lower risk of obesity [3-10]. Moreover, GTCs are involved in modulating fat metabolism $[3,5,6,8,11-13]$, but the mechanisms are not clear.

One family of transcription factors critical to fat metabolism are peroxisome proliferator-activated receptors (PPARs) (PPAR $\alpha, \gamma$, and $\delta$ ), which are ligand-activated transcription factors in the nuclear receptor superfamily [14]. Each PPAR

$\dagger$ Contributed equally to this work

*Corresponding author (email: zhaobl@ sun5.ibp.ac.cn) displays a tissue-selective expression pattern and has a distinct role in lipid metabolism [15,16]. PPAR $\alpha$, mainly expressed in liver, possibly mediates the hypotriglyceridemic effect of fibrates by inducing mitochondrial and peroxisomal $\beta$-oxidation in liver, kidney, heart, and muscle, and by decreasing the plasma concentration of triacylglycerol-rich lipoproteins [17]. PPAR $\gamma$ is one of the central regulators of adipogenesis that promotes lipid storage in adipocytes [15]. PPAR $\delta$, mainly expressed in brown adipose tissue (BAT) and muscle, specifically induces the expression of genes required for fatty acid oxidation and energy dissipation, which lead to the improvement of lipid profiles and the reduction of adiposity [16]. Increasing the levels of PPAR $\delta$ in white adipose tissue (WAT) is suggested as a potential strategy to treat obesity [18].

Green tea components have been shown to affect PPAR signaling pathways. Lee et al. [19] reported that green tea 
and its main component, epigallocatechin gallate (EGCG), increased the activation of PPAR $\alpha$. Tea catechins were also found to suppress adipocyte differentiation accompanied by down-regulation of PPAR $\gamma$ and CCAAT-enhancer-binding protein $(\mathrm{C} / \mathrm{EBP} \alpha)$ [5]. Moreover, Ashida et al. [4] reported that green tea suppressed the expression of PPAR $\gamma$, decreased the translocation of glucose transporter 4 (GLUT4), and decreased the activation of sterol regulatory element binding protein-1 in adipose tissue. Therefore, PPARs may be involved in the anti-obesity effects of green tea but no clear mechanisms have been proposed. In this study, the roles of PPARs and related pathways in the anti-obesity mechanisms of GTCs were investigated using a high-fat (HF) diet-induced obesity rat model.

\section{Materials and methods}

\subsection{Animals}

Male Sprague-Dawley rats, 30 days old weighing 180-200 g, were purchased from the Vital Active Company (Beijing, China) and were housed in a specific parasite free environment at $22^{\circ} \mathrm{C}$ with a 12 -h light-dark cycle. All rats were fed standard chow diets for $3 \mathrm{~d}$ to adapt. Rats were then randomly divided into two groups. One group was fed the regular chow diet; the other was fed a high-fat diet (15\% saturated fat, $1 \%$ cholesterol, $84 \%$ chow diet). The rats were weighed $30 \mathrm{~d}$ after starting the diet to determine increases in body weight induced by the high-fat diet. Rats fed the high-fat (HF) or chow (CHOW) diet were each randomly divided into two groups (chow diet groups 1 and 2 and high-fat diet groups 1 and 2). Each of the four groups had 10 rats. Group 1 of the chow diet group and group 1 of the high-fat diet were selected as the GTC feeding groups. Rats in the GTC feeding groups were gavaged GTCs at $100 \mathrm{mg}$ $\mathrm{kg}^{-1}$ (body weight) $\mathrm{d}^{-1}$. Body weight and food consumption of the rats were recorded every day. The rats were sacrificed 30 or $45 \mathrm{~d}$ after GTC feeding, and the liver and adipose tissue were collected and frozen at $-75^{\circ} \mathrm{C}$. GTCs used in this study were $98 \%$ pure. They contained $50 \%$ (-)-epigallocatechin gallate (EGCG), 22\% (-)-epicatechin gallate (ECG), $18 \%$ (-)-epigallocatechin (EGC), and 10\% (-)-epicatechin
(EC) (analyzed by HPLC). GTCs were provided by Fullgreen Biology Technology Co. Ltd. (Mianyang, Sichuan, China).

\subsection{Measurement of triglyceride content}

Triglycerides (TGs) were assayed according to Shimabukuro et al. [20] with modification. In brief, $50 \mathrm{mg}$ of liver tissue was homogenized in $1 \mathrm{~mL}$ of lysis buffer with the addition of 5\% (v/v) Triton X-100 to ensure homogenous lipid distribution. TG contents were measured using a colorimetric assay that quantifies the glycerol contents of the samples [20]. Serum TGs were analyzed in a Hitachi 2000 autoanalyzer. Protein concentration was measured with a BCA assay kit (Pierce, USA).

\subsection{RT-PCR}

Total RNA was extracted from adipose tissues with TRIzol Reagent (Invitrogen, USA). The total RNA ( $2 \mu \mathrm{g})$ was reverse transcribed using SuperScript First-Strand Synthesis System (Invitrogen, USA). Primers were designed according to the sequences in GenBank as listed in Table 1.

A standard $25 \mu \mathrm{L}$ PCR with $2 \mu \mathrm{L}$ of the reverse transcriptase was performed using the following parameters: $95^{\circ} \mathrm{C}, 40$ $\mathrm{s}$, annealing temperature, $40 \mathrm{~s}, 72^{\circ} \mathrm{C}, 45 \mathrm{~s}$, for 27 cycles with TaKaRa Ex Taq Hot Start Version (TaKaRa, Japan) in an MJ Research PTC-200 Peltier Thermal Cycler. The PCR reaction products $(10 \mu \mathrm{L})$ were separated on $2 \%$ agarose gels by electrophoresis. Densitometric quantification of the band intensities was analyzed using NIH Image J software.

\subsection{Western blot}

Rat adipose tissue samples were homogenized in a buffer containing $100 \mathrm{mmol} \mathrm{L}^{-1} \mathrm{NaCl}, 10 \mathrm{mmol} \mathrm{L}^{-1}$ TrisCl, $1 \mathrm{mmol} \mathrm{L}^{-1}$ EDTA, pH 8.0, $1 \mu \mathrm{g} \mathrm{mL}^{-1}$ aprotinin, $100 \mu \mathrm{g}$ $\mathrm{mL}^{-1}$ phenylmethylsulfonyl fluoride (PMSF), and $10 \mu \mathrm{g}$ $\mathrm{mL}^{-1}$ leupeptin. Tissue was then centrifuged at $12000 \times \mathrm{g}$ for $5 \mathrm{~min}$ at $4^{\circ} \mathrm{C}$ and the supernatants were collected. Protein concentrations were determined with a BCA assay kit (Pierce, USA). Soluble lysates were subjected to $10 \%$ SDS-PAGE.

Table 1 Primers used for RT-PCR ${ }^{\text {a)s }}$

\begin{tabular}{cllcr}
\hline Gene & \multicolumn{1}{c}{ Sense primer $\left(5^{\prime}-3^{\prime}\right)$} & \multicolumn{1}{c}{ Anti-sense primer $\left(5^{\prime}-3^{\prime}\right)$} & Temperature $\left({ }^{\circ} \mathrm{C}\right)$ & GenBank accession No. \\
\hline$\beta$-actin & TACAACCTCCTTGCAGCTCC & GGATCTTCATGAGGTAGTCAGTC & 58 & J00691 \\
AOX & GCCCTCAGCTATGGTATTAC & AGGAACTGCTCTCACAATGC & 63 & J02752 \\
CPT1 & TATGTGAGGATGCTGCTTCC & CTCGGAGAGCTAAGCTTGT & 64 & L07736 \\
UCP-1 & CTCACCTTTGAGCTCCTC & CTGATTTGCCTCTGGATG & 58 & M11814 \\
\hline
\end{tabular}

a) AOX, acyl-CoA oxidase; CPT1, carnitine palmitoyltransferase-1; UCP-1, uncoupling protein-1. 
Proteins were then transferred to nitrocellulose membranes and blocked with $5 \%$ non-fat milk/TBST for $1 \mathrm{~h}$ at room temperature. Membranes were incubated with primary antibodies against PPAR $\delta(1: 500)$, PPAR $\alpha(1: 500)$, PPAR- $\gamma$ $(1: 1000)$, and $\beta$-Actin $(1: 500)$. The films were scanned and densitometry of the immunoblot bands was quantitated using NIH Image J densitometry software.

\subsection{Thiobarbituric acid reactive substances (TBARS)}

The level of lipid peroxidation of liver was measured by determining TBARS. The liver homogenates $(50 \mu \mathrm{L})$ were mixed with $1 \mathrm{~mL} 20 \%(\mathrm{w} / \mathrm{v})$ trichloroacetic acid, $0.8 \mathrm{~mL}$ double distilled water, $1 \mathrm{~mL} 0.67 \%$ (w/v) 2-thiobarbituric acid, and $0.1 \mathrm{~mL} 0.2 \%(\mathrm{w} / \mathrm{v})$ butylated hydroxytoluene. After vortexing, the mixtures were incubated for $60 \mathrm{~min}$ in boiling water. TBARS were extracted with $3 \mathrm{~mL}$ of $n$-butanol and centrifuged at $4400 \times g$ for $10 \mathrm{~min}$. The absorption of the butanol layer was then measured at $532 \mathrm{~nm}$. Values are expressed in nmol of MDA $\mathrm{g}^{-1}$ of wet tissue. In the assay, 1,1,3,3-tetraethoxypropane was used as MDA standard.

\subsection{Statistics}

Results are presented as means with standard errors. Statistical analysis was performed using ORIGIN. Differences between the groups were established using the least significant difference (LSD) test. Significance was assessed at the $P<0.05$ level.

\section{Results}

\subsection{GTCs have anti-obesity and hypolipidemic effects}

The rats fed a high-fat diet had significantly more weight gain than rats fed a chow diet, and GTCs significantly decreased body weight (Figure 1A). GTC administration caused significant body weight reduction (about $9.4 \%$ and $6.3 \%$ compared to corresponding control groups) in both the $\mathrm{HF}$ and $\mathrm{CHOW}$ groups within $30 \mathrm{~d}$ (Figure 1B). This effect became more obvious (about $11.8 \%$ and $8.2 \%$ ) after $45 \mathrm{~d}$ of GTC feeding (Figure 1C). GTCs also significantly reduced the ratios of liver to body weight and the TG contents in the livers and blood plasma (Figure 1D-G). Moreover, lipid peroxidation was decreased in the livers of rats fed GTCs (Figure 1H).

\subsection{GTCs have opposite effects on PPAR $\gamma$ expression in SWAT and VWAT}

PPAR $\gamma$ is one of the central regulators of adipogenesis that promotes lipid storage in adipocytes [14]. GTCs increased the PPAR $\gamma$ level in subcutaneous white adipose tissue (SWAT) in both the HF (about 50.2\%) and CHOW (about $39.8 \%$ ) groups (Figure 2A and A1). However, GTCs decreased the levels of PPAR $\gamma$ in visceral (mesentery) white adipose tissue (VWAT) in both the HF (about 23.1\%) and CHOW (about 18.7\%) groups (Figure 2B and B1).

\subsection{GTCs up-regulate the expression of PPAR $\delta$ and its down-stream genes in adipose tissues}

In contrast to PPAR $\gamma, \operatorname{PPAR} \delta$ stimulates $\beta$-oxidation of fat and utilization of triglycerides [16]. Our results showed that administration of GTCs consistently increased PPAR $\delta$ levels in all types of adipose tissues tested. For BAT, PPAR $\delta$ level increased about $23.2 \%$ in the HF group and $19.7 \%$ in the CHOW group. For SWAT, PPAR $\delta$ level increased about $80.1 \%$ in the HF group and $39.6 \%$ in the CHOW group. For VWAT, PPAR $\delta$ level increased about $52.4 \%$ in the $\mathrm{HF}$ group and $37.3 \%$ in the $\mathrm{CHOW}$ group (Figure $3 \mathrm{~A}$ and A1). The expression of UCP-1, a marker for BAT, was also increased in SWAT and VWAT (Figure 3B and B1) after GTC treatment. To further examine the effect of GTCs on PPAR $\delta$-mediated signaling, we studied the expression of the PPAR $\delta$ down-stream target genes, carnitine palmitoyltransferase-1 (CPT1), acyl-CoA oxidase (AOX), and UCP-1, in BAT. We found that GTCs significantly increased the expression of CPT1, AOX, and UCP-1. For CPT1, expression increased about $27.4 \%$ in the $\mathrm{HF}$ group and $50.8 \%$ in the $\mathrm{CHOW}$ group (Figure $3 \mathrm{C}$ and $\mathrm{C} 1$ ). For AOX, expression increased about $42.7 \%$ in the $\mathrm{HF}$ group and $62.9 \%$ in the $\mathrm{CHOW}$ group (Figure $3 \mathrm{C}$ and $\mathrm{C} 2$ ). For UCP-1, expression increased about $19.7 \%$ in the $\mathrm{HF}$ group and $21.1 \%$ in the $\mathrm{CHOW}$ group (Figure $3 \mathrm{C}$ and $\mathrm{C} 3$ ).

\section{Discussion}

In this study, we investigated the anti-obesity and hypolipidemic mechanisms of GTCs $\left(100 \mathrm{mg}^{-1} \mathrm{~kg}^{-1} \mathrm{~d}^{-1}\right)$ using a rat model of HF diet-induced obesity. Our results showed that GTCs significantly reduced the body weight of rats after $30 \mathrm{~d}$. The differences between GTCs and control groups became more obvious after GTCs were given to the rats for $45 \mathrm{~d}$, indicating more weight loss can be achieved when GTCs are given for an extended period. Weight management is difficult and even a moderate level of weight loss can be beneficial for conditions such as hypertension and T2DM [21]. Therefore, it is impressive that GTC supplementation can cause such rapid weight loss in overweight rats. The decreased body weight in the rats fed the normal chow diet and GTCs suggests that drinking green tea may be beneficial for maintaining healthy weight in the general population. GTCs also decreased blood and liver lipids, 
A

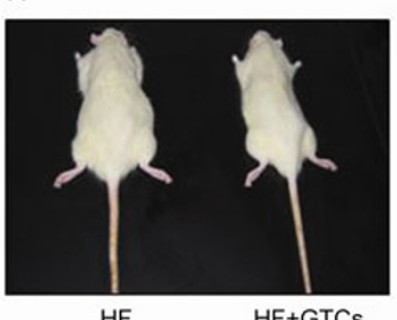

$\mathrm{D}$

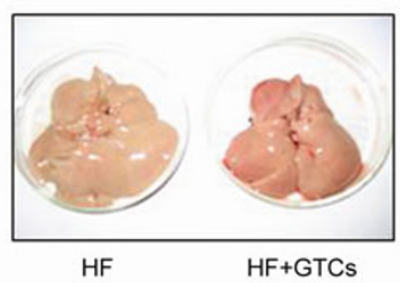

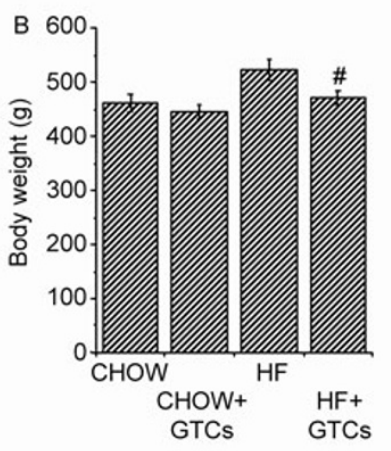
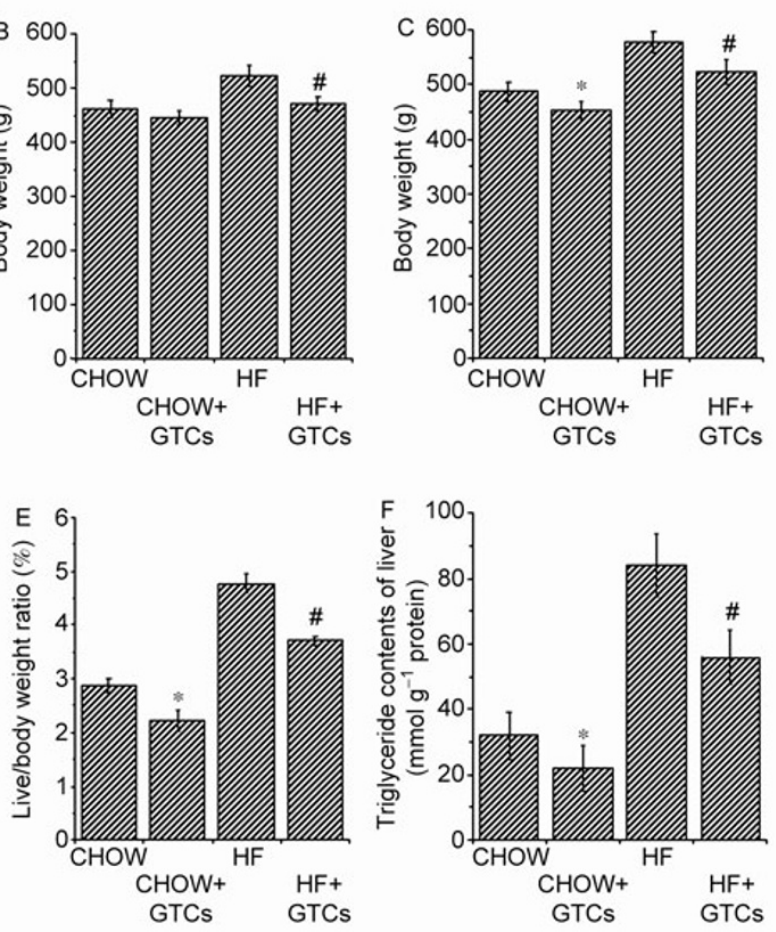
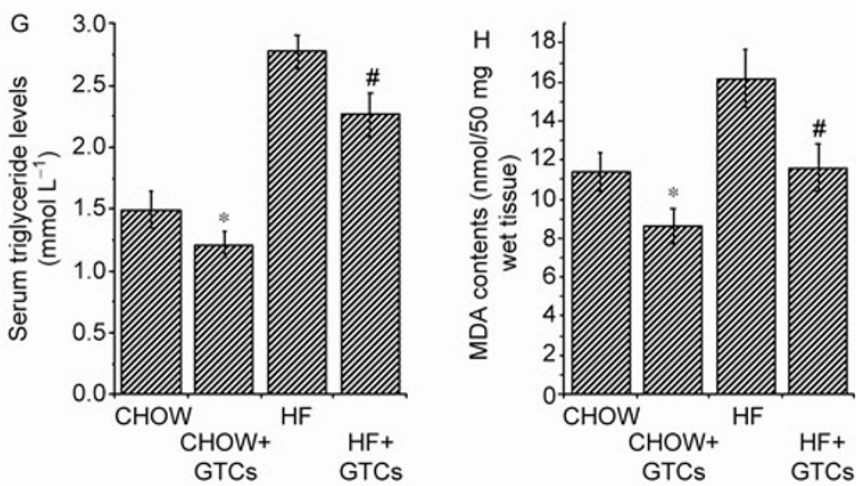

Figure 1 Anti-obesity and hypolipidemic effects of GTCs. A, Photographs of representative rat fed with or without GTCs for $45 \mathrm{~d}$ in the high-fat diet group. B and C, Body weights of CHOW (chow diet group), CHOW+GTCs (chow diet+GTCs group), HF (high-fat diet control group) and HF+GTCs (high-fat diet+GTCs group) after feeding with GTCs $100 \mathrm{mg} \mathrm{kg}^{-1}$ (body weight) $\mathrm{d}^{-1}$ or water for $30 \mathrm{~d}$ (B) or $45 \mathrm{~d}$ (C) $(n=8$; \#, $P<0.05 \mathrm{vs.} \mathrm{HF;} \mathrm{*,} P<0.05 \mathrm{vs.}$ CHOW). D, Photographs of the representative livers from rats fed with or without GTCs for $45 \mathrm{~d}$. E, GTCs reduced the liver to body weight ratios in both HF $(\#, P<0.05)$ and CHOW $(*, P<0.05)$ groups. F, GTCs reduced liver triglyceride contents in both HF $(\#, P<0.05)$ and CHOW $(*, P<0.01)$ groups. G, GTCs reduced serum triglyceride contents in both HF $(\#, P<0.01)$ and CHOW $(*, P<0.01)$ groups. H, GTCs reduced liver TBARS levels in both HF $(\#$, $P<0.01)$ and $\mathrm{CHOW}(*, P<0.01)$ groups.

which are directly related to fatty liver and atherosclerosis. By lowering the lipids stored in the liver and circulated in the blood, administration of GTCs is likely to inhibit the pathogenesis of these diseases.

Food intake by rats has been shown to be reduced by the treatment of EGCG and is proposed to contribute to the weight-loss effect of EGCG [22]. In contrast, there are also reports claiming that supplementation with green tea or
GTCs does not significantly affect food consumption $[4,23]$. These discrepancies may be caused by different supplementation methods and concentrations of GTCs. In our experiments, we found that the food intake of the rats fed GTCs was not significantly different compared to the rats fed the control diet $(P>0.1)$. Therefore, mechanisms other than a decrease in food intake were involved in the antiobesity effects of GTCs. 
A
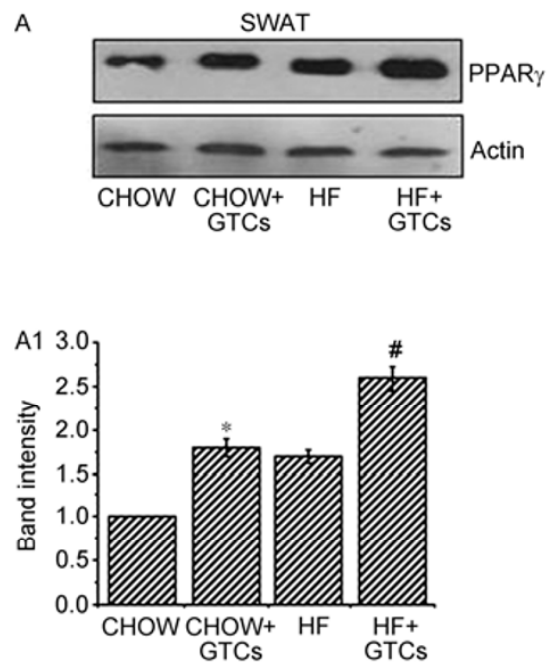

B
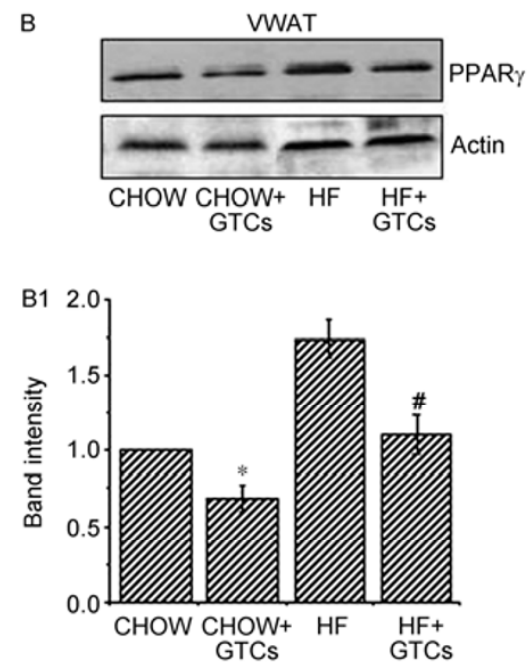

Figure 2 Effects of GTCs on the expression of PPAR $\gamma$ in subcutaneous white adipose tissue (SWAT) (A) and visceral white adipose tissue (VWAT) (B). Rats were fed different diets for $45 \mathrm{~d}$ and the adipose tissue samples were analyzed by Western blot. The intensities of the bands were quantified by densitometric analysis and the results (A1 and B1) are expressed as fold changes by normalizing the data to the values obtained from the CHOW diet group, which were set to 1.0 ( $n=6$; \#, $P<0.01$ vs. HF; *, $P<0.01$ vs. CHOW).

We found that GTCs might exert anti-obesity effects through PPAR pathways. First, our results showed that GTCs had distinct effects on the levels of PPAR $\gamma$ in different types of adipose tissue. GTCs increased the PPAR $\gamma$ level in SWAT and decreased the PPAR $\gamma$ level in VWAT. In VWAT, the decreased PPAR $\gamma$ level and decreased fat content indicated that the fat accumulation in this tissue was inhibited by the GTC treatment. In SWAT, the increased PPAR $\gamma$ level might promote the adipogenesis and lipid storage functions of SWAT. Therefore, fewer lipids would be stored in other tissues such as VWAT, liver, and blood, as shown in our results. Because improving the lipid metabolic functions of SWAT is an important strategy for the therapy of T2DM and other linked metabolic disorders [24], drinking green tea or supplementation of GTCs could be beneficial for patients with these diseases.

In addition to modulating the fat distribution in different adipose tissue, total lipid mass in the body could also be reduced by GTC treatment. We found that GTCs increased PPAR $\delta$ levels in both BAT and WAT. In addition, the expressions of PPAR $\delta$ down-stream genes such as AOX, CPT1, and UCP-1 were increased. Since BAT is the major thermogenic tissue [15], the increased protein level of PPAR $\delta$ in BAT suggests the promotion of $\beta$-oxidation in BAT by GTCs. UCP-1 is the marker gene of brown adipocytes. Therefore, the up-regulation of UCP-1 in VWAT and SWAT by GTCs suggests that the white adipocytes might have acquired the features of brown adipocytes [25]. When WAT acquires BAT features, fatty acid oxidation and ener- gy uncoupling are increased [25,26]. Taken together, GTCs increased the energy expenditure of adipocytes and reduced the total mass of lipids in the body possibly by stimulating the function of BAT and promoting BAT features in WAT.

PPAR $\alpha$ enhances lipid consumption in the liver by upregulating genes encoding enzymes in $\beta$-oxidation. Green tea but not EGCG supplementation was found to upregulate the expression of PPAR $\alpha$ in livers of rats [23,27]. In contrast, our results did not show an upregulation of PPAR $\alpha$ expression in the livers of the rats fed GTCs (data not shown). Previously, it was reported that green tea and its main component EGCG increased the activation of PPAR $\alpha$ in COS-1 cells [19]. Thus, future studies on GTCs' effects need to explore the expression and activation of PPAR $\alpha$.

Our results show that GTCs exert their anti-obesity mechanisms in part by modulating PPAR pathways. However, other mechanisms underlying the anti-obesity effects of GTCs cannot be excluded. GTCs can scavenge free radicals as antioxidants [28-31]. Moreover, oxidative stress is associated with adiposity in both human and mouse models of obesity [32-34]. Thus, GTCs may prevent obesity by restoring the antioxidative potential of the tissue. In addition, GTCs can bind to digestive enzymes, including glucosidases and lipases [35], and it is possible that they prevent the digestion and absorption of carbohydrates and lipids in the animals. This would decrease the calories absorbed even if the food intake by animals was not affected in the experiment. 

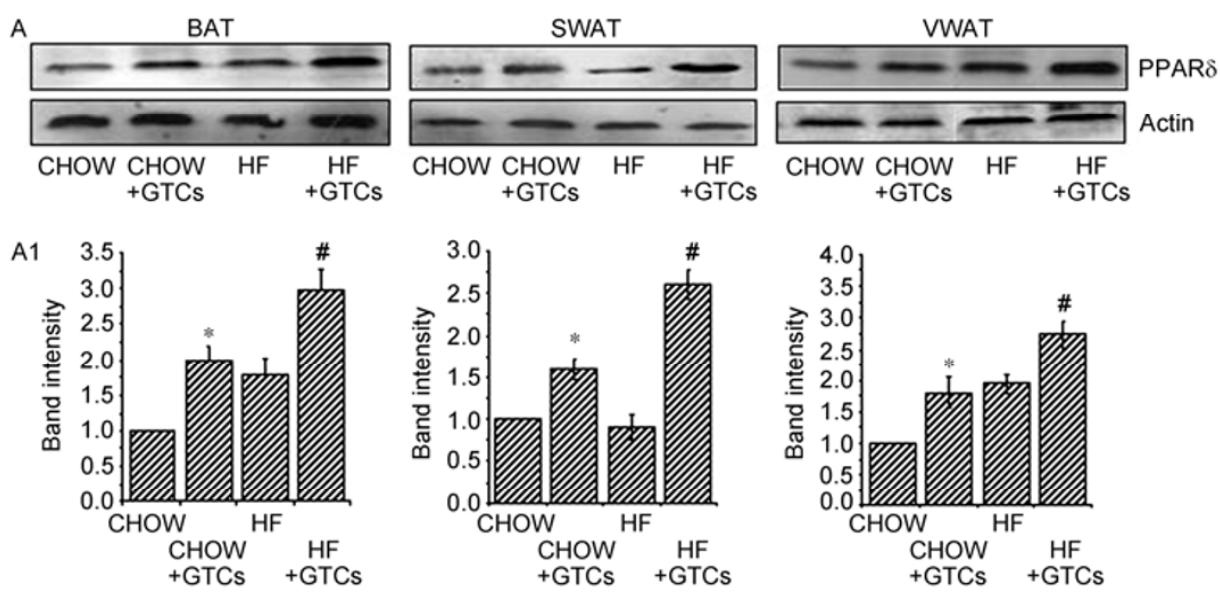

B
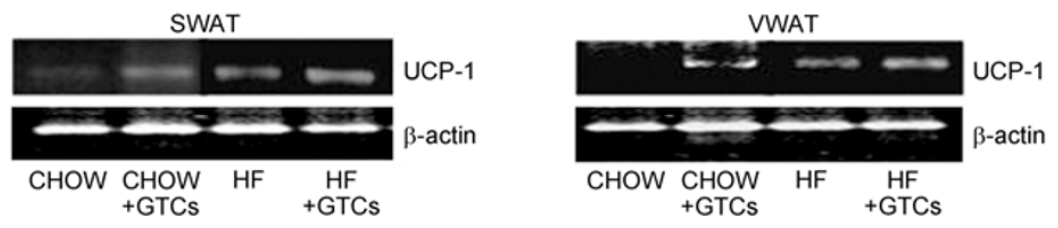

B1
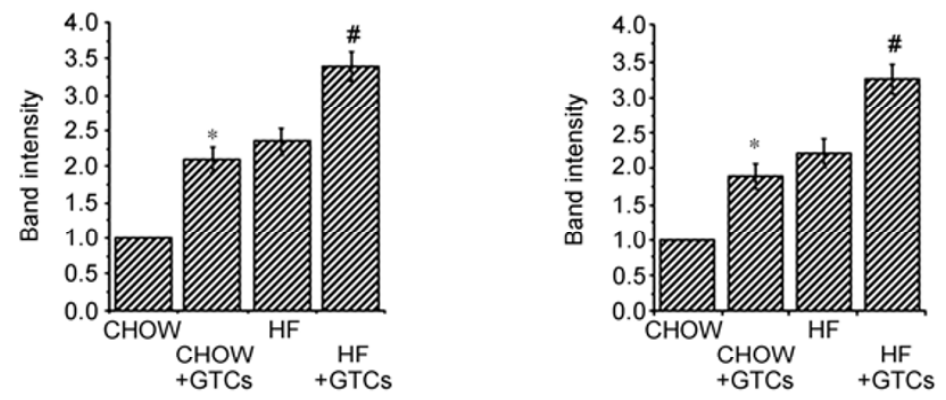

C
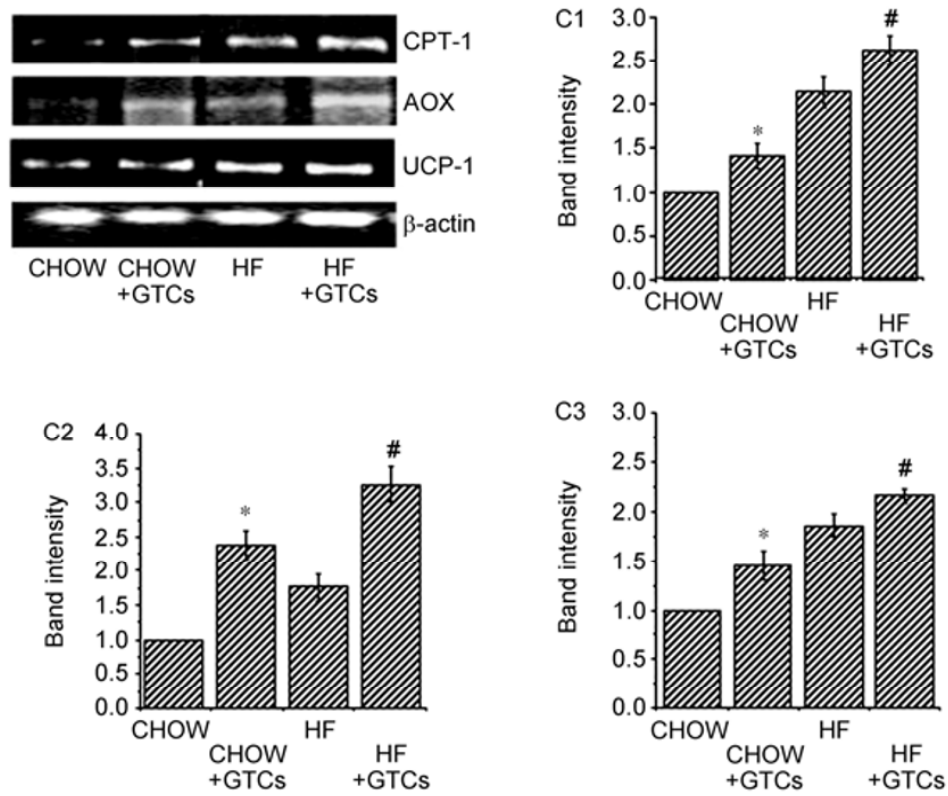

Figure 3 GTCs increased the expression of PPAR $\delta$ and activated PPAR $\delta$ pathway of beta oxidation and energy uncoupling in adipose tissues. Rats were fed different diets for $45 \mathrm{~d}$ and the adipose tissue samples were prepared. A, Levels of PPAR $\delta$ in BAT, SWAT, and VWAT analyzed by Western blot. B, mRNA levels of UCP-1 in SWAT and VWAT analyzed by RT-PCR. C, mRNA levels of CPT1, AOX, and UCP-1 in BAT analyzed by RT-PCR. The intensities of the bands were quantified by densitometric analysis and the results (A1, B1, C1, C2, C3) are expressed as fold changes by normalizing the data to the values obtained from the $\mathrm{CHOW}$ diet group, which were set to 1.0 ( $n=6$; \#, $P<0.01 \mathrm{vs.} \mathrm{HF} ;{ }^{*}, P<0.01 \mathrm{vs}$. CHOW). C1, CPT1; C2, AOX; C3, UCP-1. 
We thank Ms. Xiang Shi for her excellent technical assistance and help with animal care and administration. This work was supported by the National Natural Science Foundation of China (30170239, 30930036).

1 National Institutes of Health. Clinical guidelines on the identification, evaluation, and treatment of overweight and obesity in adults-the evidence report. Obes Res, 1998, 6(Suppl 2): 51S-209S

2 Kopelman P G. Obesity as a medical problem. Nature, 2000, 404: 635-643

3 Dulloo A G, Seydoux J, Girardier L, et al. Green tea and thermogenesis: interactions between catechin-polyphenols, caffeine and sympathetic activity. Int J Obes Relat Metab Disord, 2000, 24: 252-258

4 Ashida H, Furuyashiki T, Nagayasu H, et al. Anti-obesity actions of green tea: possible involvements in modulation of the glucose uptake system and suppression of the adipogenesis-related transcription factors. Biofactors, 2004, 22: 135-140

5 Furuyashiki T, Nagayasu H, Aoki Y, et al. Tea catechin suppresses adipocyte differentiation accompanied by down-regulation of PPARgamma2 and C/EBPalpha in 3T3-L1 cells. Biosci Biotechnol Biochem, 2004, 68: 2353-2359

6 Tokimitsu I. Effects of tea catechins on lipid metabolism and body fat accumulation. Biofactors, 2004, 22: 141-143

7 Dufresne C J, Farnworth E R. A review of latest research findings on the health promotion properties of tea. J Nutr Biochem, 2001, 12: 404-421

8 Yang $\mathrm{M}$, Wang $\mathrm{C}$, Chen $\mathrm{H}$. Green, oolong and black tea extracts modulate lipid metabolism in hyperlipidemia rats fed high-sucrose diet. J Nutr Biochem, 2001, 12: 14-20

9 Klaus S, Pultz S, Thone-Reineke C, et al. Epigallocatechin gallate attenuates diet-induced obesity in mice by decreasing energy absorption and increasing fat oxidation. Int J Obes (Lond), 2005, 29: 615623

10 Nagao T, Komine $\mathrm{Y}$, Soga S, et al. Ingestion of a tea rich in catechins leads to a reduction in body fat and malondialdehyde-modified LDL in men. Am J Clin Nutr, 2005, 81: 122-129

11 Wang X, Song K S, Guo Q X, et al. The galloyl moiety of green tea catechins is the critical structural feature to inhibit fatty-acid synthase. Biochem Pharmacol, 2003, 66: 2039-2047

12 Mori M, Hasegawa N. Superoxide dismutase activity enhanced by green tea inhibits lipid accumulation in 3T3-L1 cells. Phytother Res, 2003, 17: 566-567

13 Choo J J. Green tea reduces body fat accretion caused by high-fat diet in rats through beta-adrenoceptor activation of thermogenesis in brown adipose tissue. J Nutr Biochem, 2003, 14: 671-676

14 Kersten S, Desvergne B, Wahli W. Roles of PPARs in health and disease. Nature, 2000, 405: 421-424

15 Rosen E D, Spiegelman B M. PPARgamma: a nuclear regulator of metabolism, differentiation, and cell growth. J Biol Chem, 2001, 276: 37731-37734

16 Wang Y X, Lee C H, Tiep S, et al. Peroxisome-proliferator-activated receptor delta activates fat metabolism to prevent obesity. Cell, 2003, 113: $159-170$

17 Mukherjee R, Jow L, Croston G E, et al. Identification, characteriza- tion, and tissue distribution of human peroxisome proliferator-activated receptor (PPAR) isoforms PPARgamma2 versus PPARgamma1 and activation with retinoid $\mathrm{X}$ receptor agonists and antagonists. J Biol Chem, 1997, 272: 8071-8076

18 Walczak R, Tontonoz P. Setting fat on fire. Nat Med, 2003, 9: 13481349

19 Lee K. Transactivation of peroxisome proliferator-activated receptor alpha by green tea extracts. J Vet Sci, 2004, 5: 325-330

20 Shimabukuro M, Koyama K, Chen G, et al. Direct antidiabetic effect of leptin through triglyceride depletion of tissues. Proc Natl Acad Sci USA, 1997, 94: 4637-4641

21 Pappachan J M, Chacko E C, Arunagirinathan G, et al. Management of hypertension and diabetes in obesity: non-pharmacological measures. Int J Hypertens, 2011, 2011: 398065

22 Kao Y H, Hiipakka R A, Liao S. Modulation of endocrine systems and food intake by green tea epigallocatechin gallate. Endocrinology, 2000, 141: 980-987

23 Chen N, Bezzina R, Hinch E, et al. Green tea, black tea, and epigallocatechin modify body composition, improve glucose tolerance, and differentially alter metabolic gene expression in rats fed a high-fat diet. Nutr Res, 2009, 29: 784-793

24 DeFronzo R A. Dysfunctional fat cells, lipotoxicity and type 2 diabetes. Int J Clin Pract Suppl, 2004, 9-21

25 Spiegelman B M, Flier J S. Adipogenesis and obesity: rounding out the big picture. Cell, 1996, 87: 377-389

26 Tiraby C, Tavernier G, Lefort C, et al. Acquirement of brown fat cell features by human white adipocytes. J Biol Chem, 2003, 278: 33370_ 33376

27 Abe K, Okada N, Tanabe H, et al. Effects of chronic ingestion of catechin-rich green tea on hepatic gene expression of gluconeogenic enzymes in rats. Biomed Res, 2009, 30: 25-29

28 Zhao B L, Li X J, He R G, et al. Scavenging effect of extracts of green tea and natural antioxidants on active oxygen radicals. Cell Biophys, 1989, 14: 175-185

29 Guo Q, Zhao B, Li M, et al. Studies on protective mechanisms of four components of green tea polyphenols against lipid peroxidation in synaptosomes. Biochim Biophys Acta, 1996, 1304: 210-222

30 Guo Q, Zhao B, Shen S, et al. ESR study on the structure-antioxidant activity relationship of tea catechins and their epimers. Biochim Biophys Acta, 1999, 1427: 13-23

31 Zhao B, Guo Q, Xin W. Free radical scavenging by green tea polyphenols. Methods Enzymol, 2001, 335: 217-231

32 Furukawa S, Fujita T, Shimabukuro M, et al. Increased oxidative stress in obesity and its impact on metabolic syndrome. J Clin Invest, 2004, 114: 1752-1761

33 Bondia-Pons I, Ryan L, Martinez J A. Oxidative stress and inflammation interactions in human obesity. J Physiol Biochem, 2012, 68: 701-711

34 Keaney J F Jr., Larson M G, Vasan R S, et al. Obesity and systemic oxidative stress: clinical correlates of oxidative stress in the Framingham Study. Arterioscler Thromb Vasc Biol, 2003, 23: 434-439

35 Nakai M, Fukui Y, Asami S, et al. Inhibitory effects of oolong tea polyphenols on pancreatic lipase in vitro. J Agric Food Chem, 2005, 53: 4593-4598

Open Access This article is distributed under the terms of the Creative Commons Attribution License which permits any use, distribution, and reproduction in any medium, provided the original author(s) and source are credited. 